\title{
To Study the Prevalence of the Absence of Palmaris Longus in the Punjabi Population - An Institutional Study
}

\author{
Parminder Singh Kular ${ }^{1}$, Rajinder Garg ${ }^{2}$, Pankaj Miglani ${ }^{3}$, Sandeep Kaur ${ }^{4}$ \\ ${ }^{1}$ Associate Professor, Department of Orthopaedics, ${ }^{2}$ Professor, Department of Orthopaedics, ${ }^{3}$ Associate Professor, \\ Department of Orthopaedics, ${ }^{4}$ Professor Head, Department of Physiology, Adesh Institute of medical Sciences and Research, \\ Bathinda, India
}

Corresponding author: Sandeep Kaur, Professor Head, Department of Physiology, Adesh Institute of medical Sciences and Research, Bathinda, India

DOI: http://dx.doi.org/10.21276/ijcmsr.2019.4.3.38

How to cite this article: Parminder Singh Kular, Rajinder Garg, Pankaj Miglani, Sandeep Kaur. To study the prevalence of the absence of palmaris longus in the punjabi population - an institutional study. International Journal of Contemporary Medicine Surgery and Radiology. 2019;4(3):C176-C178.

\section{A B S T R A C T}

Introduction: The role of Palmaris longus in human beings in hand function is not important and hence can be harvested as tendon graft without any donor site morbidity. Geographical variations in the prevalence of the absence of Palmaris longus tendon are well known. This study was undertaken to know the absence of Palmaris longus in Punjabi population.

Material and methods: The study was done in six hundred medical students aged between 18 and 25 years of Adesh Institute of Medical Science and Reseach, Bathinda. Equal number of male and female students were examined for the presence or absence of the Palmaris Longus tendon using the standard Schaeffer's test. The absence of the palmaris longus was confirmed by three other tests to avoid any error.

Result: In the present study Palmaris longus showed overall absence of $11.3 \%$ in Punjabi population .Overall absence of Palmaris longus in males and females was $17.3 \%$ and $9.3 \%$ respectively .Unilateral and bilateral absence was $6 \%$ and $2 \%$ respectively in males whereas $8 \%$ and $5.3 \%$ in females. Overall absence was $5.3 \%$ and $1.6 \%$ on left and right side respectively. Conclusion: The prevalence of bilateral and unilateral absence was more common on left sided and in female subjects. This can help in planning surgeries as overall absence of Palmaris longus is Punjabi Population is less and is mostly unilateral.

Keywords: Palmaris longus, Punjabi Population, Tendon Grafts, Students, Agenesis

\section{INTODUCTION}

The palmaris longus (PL) is muscle of superficial anterior compartment of the forearm and is located between the flexor carpi radialis and the pronator teres muscles Palmaris longus basically, anchor skin and fascia of hand resist the horizontal shear force in distal direction. It also helps in tightening of palmar aponeurosis. Along with other flexor muscle it also helps in flexion of wrist joint. ${ }^{1}$

Palmaris longus is considered to be a dispensible because its absence is not associated with any functional limitation. It is therefore usually applied in reconstructive surgeries, tendon transfers, second stage tendon reconstruction, pulley reconstruction as well as tendon grafts. ${ }^{2}$ Moreover, Palmaris longus meets the important criteria of appropriate length and diameter so it also makes a nice backup in case tendon transfer is required.

Palmaris Longus has the greatest variation which may include an additional belly of the muscle, fusion with another muscle, bifurcated tendon, reversed muscle, an atypical tendon course and inserts, or multiple tendinous insertion. ${ }^{3,4}$ The most common variation of Palmaris longus is agenesis. ${ }^{5}$ If Palmaris Longus is absent the orthopaedic surgeons need to plan for alternative tendon graft like Plantaris or Gracilis for surgical procedures.

Various studies quote the prevalence of absence of Palmaris longus The data from other studies cannot be relied upon adue to variable reporting of prevalence of its absence in different population and races. ${ }^{6,7}$ The aim of the study is to know occurrence of absence in Palmaris Longus in Punjabi population. The presence of the Palmaris Longus can be determined through non -invasive and standard physical examination of the volar wrist. ${ }^{8}$ So, the present study was done on medical students of Adesh Institute of Medical Sciences and Research Bathinda as students are admitted here from different places of Punjab. The results will be useful for orthopaedic surgeons working in our population.

\section{MATERIAL AND METHODS}

The study was done on six hundred medical students of Adesh Institute of Medical Sciences and Research, Bathinda aged between 18 and 25 years. Equal number of male and female medical students who came for clinical posting in the orthopaedics were included in the study after getting approval from Ethical Committee of the Institute The students with a history of injury, operation, disease, or abnormality of the 


\begin{tabular}{|l|c|c|c|c|c|c|}
\hline Gender & Number & $\begin{array}{c}\text { Absent } \\
\text { Bilaterally }\end{array}$ & $\begin{array}{c}\text { Absent Left side } \\
\text { only }\end{array}$ & $\begin{array}{c}\text { Absent Right } \\
\text { side only }\end{array}$ & $\begin{array}{c}\text { Absent } \\
\text { Unilaterally }\end{array}$ & Overall Absent \\
\hline Male & 300 & $10(3.3 \%)$ & $12(4 \%)$ & $6(2 \%)$ & $18(6 \%)$ & $28(9.3 \%)$ \\
\hline Female & 300 & $16(5.3 \%)$ & $20(6.6 \%)$ & $4(1.3 \%)$ & $24(8 \%)$ & $40(17.3 \%)$ \\
\hline Total & 600 & $26(4.3 \%)$ & $32(5.3 \%)$ & $10(1.6 \%)$ & $42(7 \%\}$ & $68(11.3 \%)$ \\
\hline \multicolumn{7}{|r|}{ Table-1: Showing side distribution and overall of Prevalence of absence of Palmaris Longus } \\
\hline
\end{tabular}

upper limb were excluded from the study.

For the assessment of the Palmaris Longus tendon, initially each subject was made to do the standard Schaeffer's test by opposing the thumb and little finger with flexion of the wrist and the forearm in supination. ${ }^{9}$ The presence of Palmaris longus was confirmed by the protuberance under the skin which can be palpated and seen at inspection while doing the flexion of the wrist. In case of doubt in the presence or absence of Palmaris longus, then an extending force was applied to the hand. If the tendon was still not visualized or palpable, additional three tests were done to confirm the absence.

Mishra's test 1;passive hyperextension of metacarpophalangeal joints done while examining with the subject opposing itdoing flexion ${ }^{10}$ Figure -1].

Mishra's test II:the subjects abducts the thumb against resistance with wrist in slight palmar flexion ${ }^{10}$ [Figure-2].

Pushpakumars two finger sign:[Fig-3] The subject extends the index and middle finger and wrist and other fingers flexed and thumb opposed ${ }^{11}$ [Figure-3].

Absence of any protuberance beneath the skin in the distal forearm and inability to palpate, is taken as the agenesis of the Palmaris Longus tendon. The presence or absence of the Palmaris Longus tendon was recorded on both right and left side and for males and females seperately.Data was recorded in MS Office Excel and percentage scores were used to analyse unilateral and bilateral agenesis in both males and females and to calculate overall agenesis in population .

\section{RESULTS}

The study was done in six hundred medical students showed presence of Palmaris longus in 90\% males and $86.6 \%$ females. Overall absence of Palmaris longus was observed 11.3\%

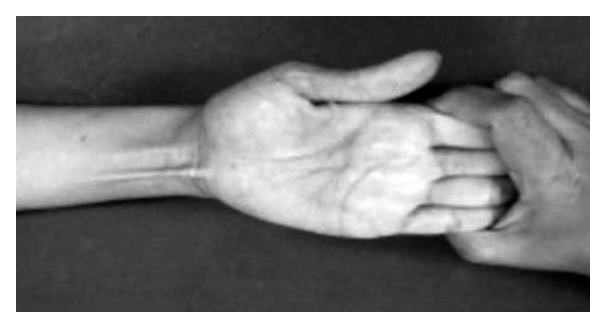

Figure-1: Mishra's test 1

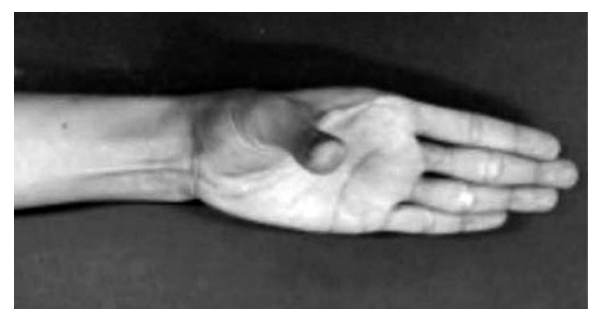

Figure-2: Mishra's test II

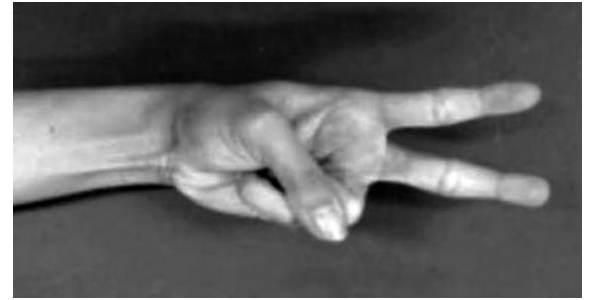

Figure-3: Pushpakumars two finger sign

in Punjabi Population with $17.3 \%$ and $9.3 \%$ in males and females respectively. Both unilateral and bilateral absence was more in females as compared to males . Overall absence of Palmaris Longus was more on left side $5.3 \%$ as compared to right side $1.6 \%$.[Table-1]

\section{DISCUSSION}

It is seen absence of Palmaris Longus varies depending on the population. As medical students were well versed in anatomy it was easy to explain the procedure to them and get excellent subject compliance while doing the examination.

In our study overall absence irrespective of gender and side was found to be $11.3 \%$ in Punjabi population which was similar to study done in Indian Population ${ }^{15}$ but this prevalence was half that in Andhra Population of India. ${ }^{14}$ If compared with populations of the world, similar prevalence was seen in Malayasian population ${ }^{12}$ and much higher values in Nigerian population and of Brazil population. ${ }^{16,17}$ This reaffirms that the prevalence of absence of the palmaris longus muscle shows a marked variation in different populations

In our study absence was reported unilaterally in $7 \%$ of the students and bilaterally in $4.3 \%$ of the students which was similar to studies done in Malaysia and Kathmandu with which also showed high prevalence of absence of Palmaris longus unilaterally than bilaterally. ${ }^{12,13} \mathrm{~A}$ study done in Andhra population of India also showed absence of unilateral agenesis more frequent than bilateral agenesis although unilateral frequency was quite high $70.5 \%$ as compared to other populations..$^{14}$

The literature on different races and population have showed left sided agenesis was more common in female than in male ${ }^{16,17}$ as in our present study in which unilateral absence was $5.3 \%$ on left side and $1.6 \%$ on right side. Controversial findings have been reported by some researchers where right sided agenesis was found to be more than left sided agenesis in male. ${ }^{18,19}$

In the present study unilateral agenesis(8\%) and bilateral agenesis(5.3\%) was more in females as compared to males. Similar results of Palmaris longus agenesis more in females than males although no significant difference were seen in other studies. ${ }^{20,21}$

Although investigating in huge population with clinical 
testing may be feasible way of evaluating the presence or absence of Palmaris longus For more accurate confirmation of Palmaris longus agenesis one of the suggested ultrasonography or magnetic resonance imaging ${ }^{22}$ techniques can be used for future studies in small population sample.

\section{CONCLUSION}

In the present study Palmaris longus absence in Punjabi population with clinical tests showed an overall absence of $11.3 \%$ which will help orthopaedic surgeons of the region for planning tendon grafts and reconstructive surgeries. The present investigation adds to the literature and reaffirms with the other studies that the agenesis is race and population dependent.

\section{REFRENCES}

1. Ioannis D, Anastasios K, Konstantinos N, Lazaros K, Georgios N. Palmaris Longus Muscle's Prevalence in Different Nations and Interesting Anatomical Variations: Review of the Literature. J Clin Med Res. 2015;7(1): 825-830.

2. Alshaham AA, Mular P, KhadimJ. Anamolous V Shaped Palmaris Longus Tendon: Two Cases. Journal of Medical Cases 2010; 1(2): 68-70

3. Olewnik et al. Anatomical variations of the palmaris longus muscle including its relation to the median nerve - a proposal for a new classification BMC Musculoskeletal Disorders 2017;18 (3):539

4. Bernardes A, Melo C, Pinheiro S. A combined variation of Palmaris longus and Flexor digitorum superficialis: Case report and review of literature. Morphologie. 2016;100 (6):245-9.

5. Thompson NW, Mockford BJ, Cran GWAbsence of the palmaris longus muscle: a population study. The Ulster Med J. 2001; 70 (1): 22 - 24.

6. Machado AB, DiDio LJ. Frequency of the musculus Palmaris longus studied in vivo in some Amazon Indians. Am J Phys Anthropol. 1967;27 (4):11-20.

7. Reimann AF, Daseler EH, Anson BJ, Beaton LE. The palmaris longus muscle and tendon. A study of 1600 extremities. Anat Rec. 1944;89 (3):495-505.

8. Eric M, Koprivcicc I, Vucinic N Prevalence of the palmaris longus in relation to the hand dominance. Surgical and Radiologic Anatomy. 2011;33(6):481-484.

9. Schaeffer JP. On the variations of the palmaris longus muscle. Anat Rec 1909;3 (1):275-8

10. Mishra S. Alternative tests in demonstrating the presence of Palmaris longus. Indian J Plast Surg. 2001;34 (3):12

11. Pushpakumar SB, Hanson RP, Carroll S. The 'two finger' sign. Agarwal: Absence of palmaris longus tendon in Indian population Clinical examination of palmaris longus (PL) tendon. Br J Plast Surg. 2004;57 (6):184185

12. SA Roohi, L Choon-Sian, A Shalimar, GH Tan, AS Naicker, M Med Rehab Study on the Absence of Palmaris Longus in a Multiracial Population Malaysian Orthopaedic Journal. 2007;1 (1):26-28.

13. Dilip K. Mehta, Rajeev Shrestha, Dil I. Mansur1, Shobha Shah, Retina Shrestha and Binod Timalsina Prevalence of the absence of Palmaris longus muscle in the students of Kathmandu University International Journal of Biomedical Research 2015; 6(12): 953-957.

14. K. Devi Sankar, P. Sharmila Bhanu, Susan P. John Incidence of agenesis of palmaris longus in the Andhra population of India Indian Journal of Plastic Surgery 2011: 44 (1):134-138.

15. Pawan Agarwal. Absence of the palmaris longus tendon in Indian population .Indian J Orthop. 2010; 44(2): 212-215.

16. Morais MA, Gomes MS, Helrigle C, Malysz T. Prevalence of agenesis of the Palmaris longus muscle in Brazil and its clinics correlation. Journal of Morphological Sciences 2012; 29(4): 238-242.

17. Adejuwon SA, Salawu OT, Ajibola B. Agenesis of Palmaris Longus Muscle in Selected Population of School Children in Nigeria. International Journal of Morphology 2012; 30(4): 1593-1596.

18. Hussain FN, Hasan T. Prevalence of congenital absence of PL tendon in young HJizani population of Saudi Arabia: A cross sectional study. Pakistan Journal of Medical Sciences 2012; 28(5): 865-869.

19. Karim SH, Ali-Taee IS. Variation in the Palmaris longus tendon in Sulaimani population. Journal of Sulaimani Medical College 2012; 2(1): 67-72.

20. Ceyhan O, Mavt A. Distribution of agenesis of the palmaris longus muscle in 12-18 years old age groups. Indian J Med Sci 1997;51 (4):156-60.

21. Thompson NW, Mockford BF, Cran GW. Absence of the palmaris longus muscle: A population study. Ulster Med J 2001;70 (6):22-24.

22. Schuurman AH, Van Gils AP. Reversed palmaris longus muscle on MRI: Report of four cases. Eur Radiol 2000;10 (3): 1242-1244.

Source of Support: Nil; Conflict of Interest: None

Submitted: 13-08-2019; Accepted: 10-09-2019; Published online: 17-09-2019 